\section{Differential expression of KIF11 in cancers of the breast.}
Shahan Mamoor, MS ${ }^{1}$
$3 \quad$ shahanmamoor@gmail.com
East Islip, NY 11730
Breast cancer affects women at relatively high frequency ${ }^{1}$. We mined published microarray datasets ${ }^{2,3}$ to determine in an unbiased fashion and at the systems level genes most differentially expressed in the primary tumors of patients with breast cancer. We report here significant differential expression of the gene encoding the kinesin family member 11, KIF11, when comparing primary tumors of the breast to the tissue of origin, the normal breast. KIF11 was also differentially expressed in the tumor cells of patients with triple negative breast cancer. KIF11 mRNA was present at significantly higher quantities in tumors of the breast as compared to normal breast tissue. Analysis of human survival data revealed that expression of KIF11 in primary tumors of the breast was correlated with overall survival in patients with basal and luminal A subtype cancer, but in a contrary manner. KIF11 may be of relevance to initiation, maintenance or progression of cancers of the female breast.

Keywords: breast cancer, KIF11, kinesin family member 11, systems biology of breast cancer, targeted therapeutics in breast cancer. 
Invasive breast cancer is diagnosed in over a quarter of a million women in the United States each year $^{1}$ and in 2018 , breast cancer was the leading cause of cancer death in women worldwide ${ }^{4}$. While patients with localized breast cancer are provided a 99\% 5-year survival rate, patients with regional breast cancer, cancer that has spread to lymph nodes or nearby structures, are provided an $86 \% 5$-year survival rate $^{5,6}$. Patients with metastasis to distant sites, like the brain, are provided a $27 \% 5$-year survival rate ${ }^{5,6}$. Understanding how primary tumors are most transcriptionally different from the tissue from which they originate, the breast, can facilitate development of novel diagnostic and therapeutics to promote early detection and enhanced treatment, and contribute to efforts to prevent progression to metastatic stages. We mined published microarray data ${ }^{2,3}$ to understand at the transcriptome level and in an unbiased fashion genes most differentially expressed in primary tumors of the breast as compared to normal breast tissue. The kinesin family member 11 emerged as among the most differentially expressed genes in cancer of the female breast.

\section{Methods}

We utilized datasets GSE109169² and GSE $38959^{3}$ for this differential gene expression analysis of female breast cancer. GSE109169 was generated using Affymetrix Human Exon 1.0 ST Array technology with $n=25$ normal breast tissue and $n=25$ tumors of the breast; analysis was performed using platform GPL5175. The tissues from this first dataset are paired tissues ( 25 tumors matching 25 breast tissues from 25 patients). GSE38959 was generated using Agilent-014850 Whole Human Genome Microarray 4x44K G4112F technology with $n=13$ samples of normal mammary gland ductal cells and $n=30$ samples of tumor cells from patients with triple negative breast cancer; analysis was performed using platform GPL4133. The Benjamini and Hochberg method of p-value adjustment was used for ranking of differential expression but raw $p$-values were used to assess statistical significance of global differential expression. Log-transformation of data was auto-detected, and the NCBI generated category of platform annotation was used. A statistical test was performed to evaluate whether KIF11 expression was significantly different between primary breast tumors and breast tissue using a two-tailed t-test. For Kaplan-Meier survival analysis, we used the Kaplan-Meier plotter online tool ${ }^{7}$ for correlation of KIF11 mRNA expression levels with overall survival (OS) in $n=404$ patients with basal subtype cancer, $n=794$ patients with luminal A subtype cancer, $n=515$ patients with luminal B subtype cancer, and $n=166$ patients with HER2+ cancer.

\section{Results}

We performed discovery of genes associated with breast cancer in females by mining two independently published microarray datasets ${ }^{2,3}$.

\section{KIF11 is differentially expressed in primary tumors of the breast.}

Comparison of 25 normal breast tissues to 25 tumors of the breast ${ }^{2}$ from patients with early-onset breast cancer revealed that the gene encoding the kinesin family member 11, encoded by the KIF11 gene, was among the genes most differentially expressed in tumors of the breast in human breast cancer (Chart 1). When sorting each of the genes expressed in tumors of the breast based on significance of difference as compared to normal breast tissue, KIF11 ranked 169 out of 19076 total transcripts, equating to $99.1 \%$ differential expression (Chart 1). Differential expression of KIF11 in female breast cancer was statistically significant (Chart $1 ; p=1.73 \mathrm{e}-13$ ).

Analysis of a second microarray datase ${ }^{3}$, here in tumor cells of patients with triple negative breast cancer as compared to normal mammary gland ductal cells, again revealed significant differential expression of KIF11 in human breast cancer (Chart 2). When sorting each of the genes expressed in the 
tumor cells of patients with triple negative breast cancer based on significance of difference as compared to normal mammary gland ductal cells, KIF11 ranked 291 out of 45015 total transcripts, equating to $99.4 \%$ differential expression (Chart 2). Differential expression of KIF11 in the tumor cells of patients with triple negative breast cancer was statistically significant (Chart $2 ; p=1.89 \mathrm{e}-10)$. These data suggested that differential expression of KIF11 was not an artifact of a single microarray dataset, nor was it associated with one type of breast cancer, rather a general feature of cancers of the breast.

\section{KIF11 is expressed at significantly higher levels in breast tumors as compared to the breast.}

We obtained exact mRNA expression levels for KIF11 from the breast and from breast tumors ${ }^{2}$ to understand the magnitude and direction of KIF11 expression change. KIF11 was expressed at higher levels in tumors of the breast as compared to normal breast tissue (Figure 1). Increased expression of KIF11 in primary breast tumors was statistically significant (Figure 1: $p<0.0001$ ). KIF11 was expressed at $3.96 \pm 0.43$ arbitrary units (AU) in normal breast tissue, while it was expressed at $5.95 \pm 0.94 \mathrm{AU}$ in tumors of the breast. We calculated a mean fold change of 1.50 in KIF11 mRNA levels when comparing primary tumors of the breast to normal breast tissues.

\section{KIF11 expression correlates with survival outcomes in basal and luminal A human breast cancer.}

We performed Kaplan-Meier survival analysis in an attempt to correlate KIF11 mRNA expression levels with survival outcomes in patients with breast cancer, evaluating correlations between molecular subtype and overall survival. We observed a statistically significant correlation between KIF11 expression and overall survival (OS) in patients with luminal A subtype breast cancer (Figure 2; log rank $p$-value: 0.00015 for overall survival, hazard ratio: 1.86 (1.34-2.57) (Fig. 2)). KIF11 mRNA levels were a negative prognostic indicator in luminal A subtype breast cancer patients. Median OS was 175.46 months for luminal A patients with low tumor expression of KIF11 while median OS was 95.04 months for luminal A patients with high tumor expression of KIF11 (Chart 3). We also observed a correlation between KIF11 expression and overall survival (OS) in patients with basal subtype breast cancer which approached statistical significance (Figure 2; log rank $p$-value: 0.087 for overall survival, hazard ratio: 0.72 (0.49-1.05) (Fig. 2)). KIF11 mRNA levels were a positive prognostic indicator in basal subtype breast cancer patients. Median OS was 52.92 months for basal subtype patients with low tumor expression of KIF11 while median OS was 75.37 months for basal subtype patients with high tumor expression of KIF11 (Chart 3). KIF11 primary tumor expression was not correlated with overall survival in luminal B subtype (Figure 2; log rank $p$-value: 0.2 for OS, hazard ratio: 1.26 (0.89-1.78) (Fig. 2)), or in HER2+ breast cancer (Figure 2; log rank $p$-value: 0.91 for OS, hazard ratio: 1.03 (0.59-1.82) (Fig. 2)).

Thus, through comparative transcriptome analysis of primary tumors of the breast and normal breast tissue, we found that differential expression and up-regulation of KIF11 was among the most significant transcriptional features in primary tumors from patients with breast cancer. KIF11 expression in primary tumors of the breast was correlated with overall survival in patients with basal and luminal A subtype cancers, with high expression of KIF11 associating with superior survival in basal subtype cancer but with low expression of KIF11 associating with superior survival in luminal A subtype cancer.

\section{Discussion}

Invasive breast cancer is a medical problem with a $27 \% 5$-year survival rate for women whose disease has spread to distant sites ${ }^{5,6}$. To facilitate understanding of the basic transcriptional differences between primary tumors of the breast and the tissues from which these tumors originate, normal breast tissues, we performed comparative transcriptome analysis using two independently published microarray 


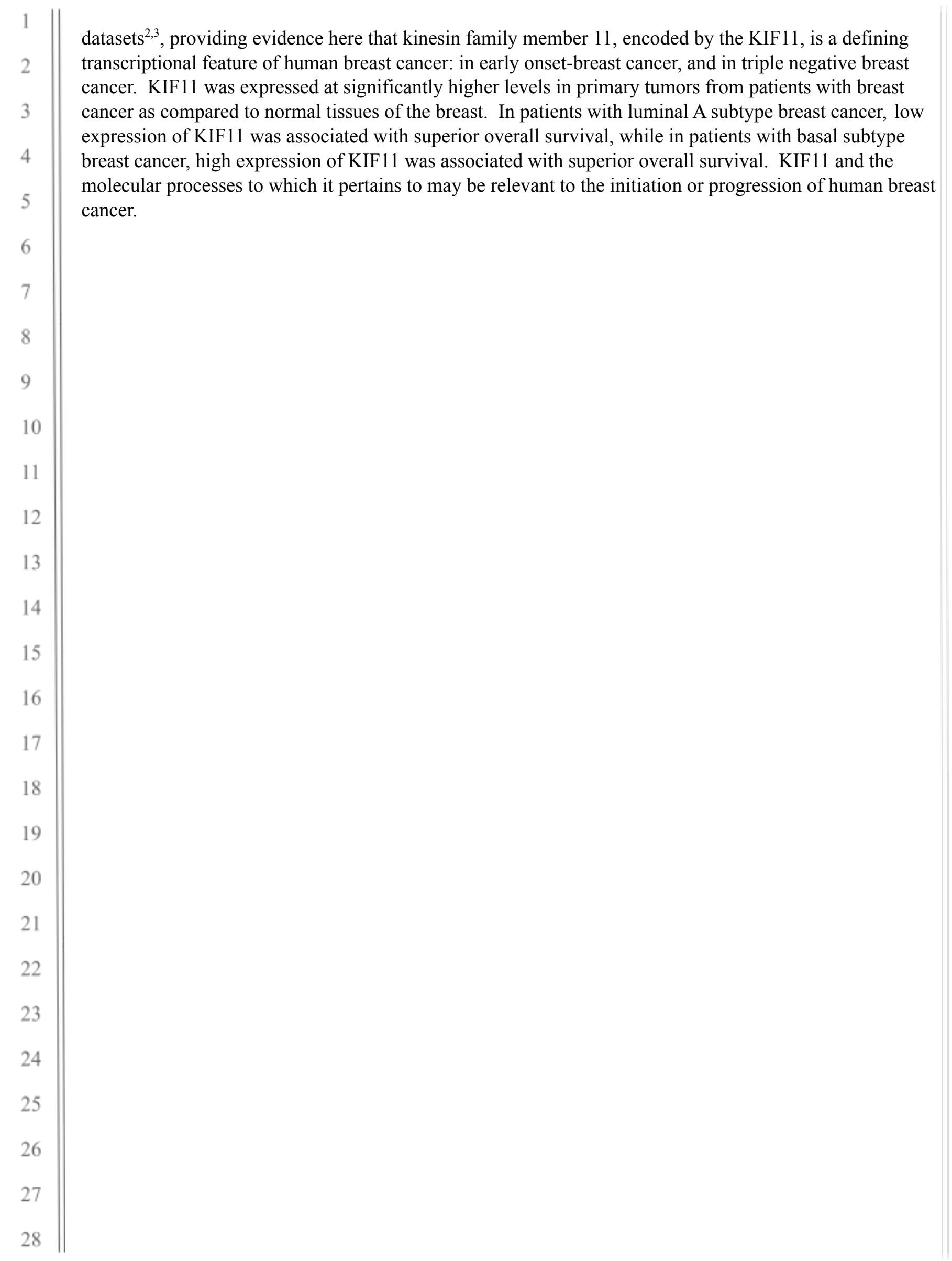




\section{References}

1. DeSantis, C.E., Ma, J., Goding Sauer, A., Newman, L.A. and Jemal, A., 2017. Breast cancer statistics, 2017, racial disparity in mortality by state. CA: a cancer journal for clinicians, 67(6), pp.439-448.

2. Chang, J.W., Kuo, W.H., Lin, C.M., Chen, W.L., Chan, S.H., Chiu, M.F., Chang, I.S., Jiang, S.S., Tsai, F.Y., Chen, C.H. and Huang, P.H., 2018. Wild-type p53 upregulates an early onset breast cancer-associated gene GAS7 to suppress metastasis via GAS7-CYFIP1- mediated signaling pathway. Oncogene, 37(30), pp.4137-4150.

3. Komatsu, M., Yoshimaru, T., Matsuo, T., Kiyotani, K., Miyoshi, Y., Tanahashi, T., Rokutan, K., Yamaguchi, R., Saito, A., Imoto, S. and Miyano, S., 2013. Molecular features of triple negative breast cancer cells by genome-wide gene expression profiling analysis. International journal of oncology, 42(2), pp.478-506.

4. Bray, F., Ferlay, J., Soerjomataram, I., Siegel, R.L., Torre, L.A. and Jemal, A., 2018. Global cancer statistics 2018: GLOBOCAN estimates of incidence and mortality worldwide for 36 cancers in 185 countries. CA: a cancer journal for clinicians, 68(6), pp.394-424.

5. ACS Cancer Facts \& Figures 2019.

https://www.cancer.net/cancer-types/breast-cancermetastatic/statistics.

6. Survival Rates for Breast Cancer. https://www.cancer.org/cancer/breast-cancer/ understanding-a-breast-cancer-diagnosis/breast-cancer-survival-rates.html

7. Györffy, B., Lanczky, A., Eklund, A.C., Denkert, C., Budczies, J., Li, Q. and Szallasi, Z., 2010. An online survival analysis tool to rapidly assess the effect of 22,277 genes on breast cancer prognosis using microarray data of 1,809 patients. Breast cancer research and treatment, 123(3), pp.725-731. 


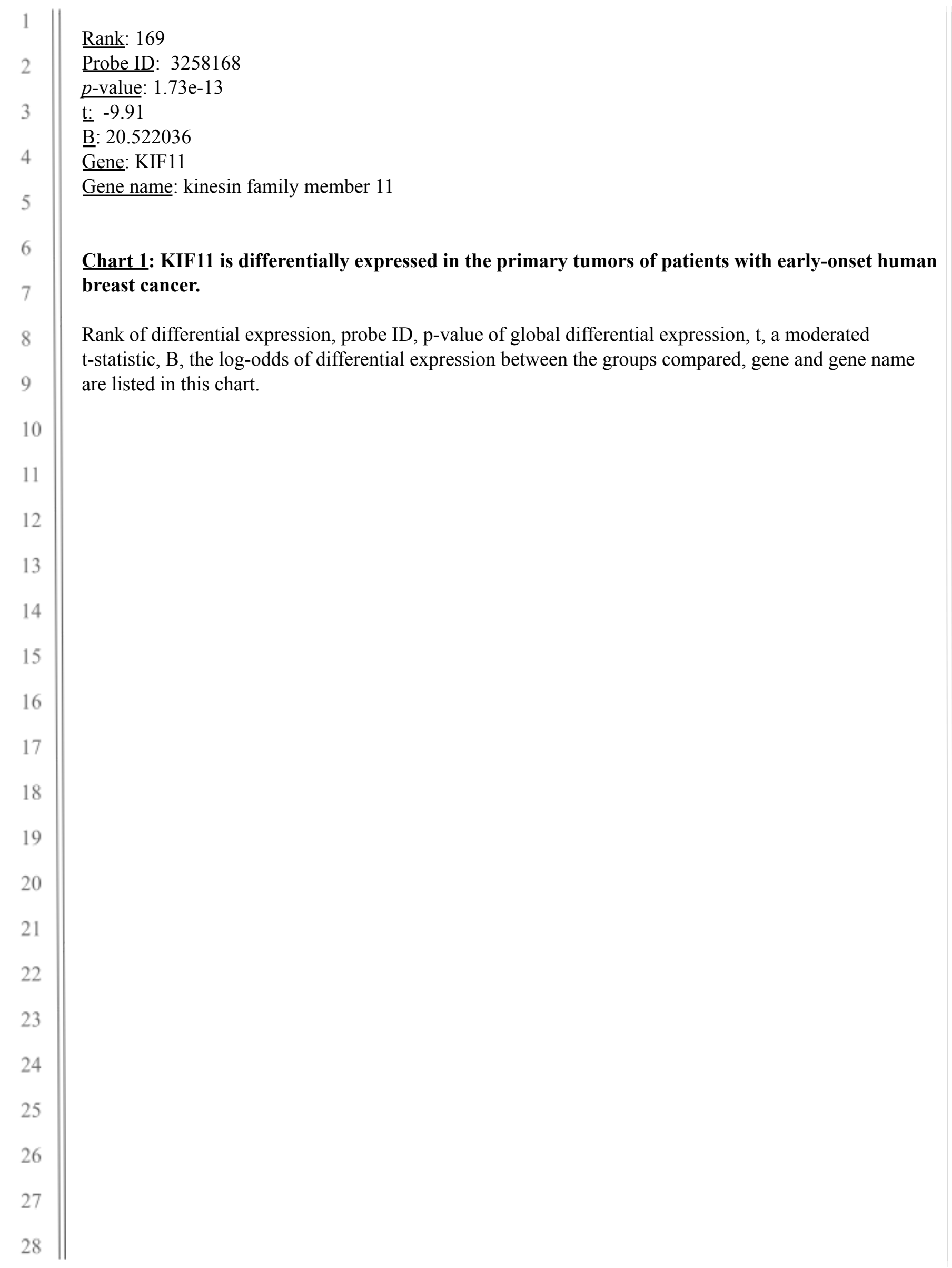




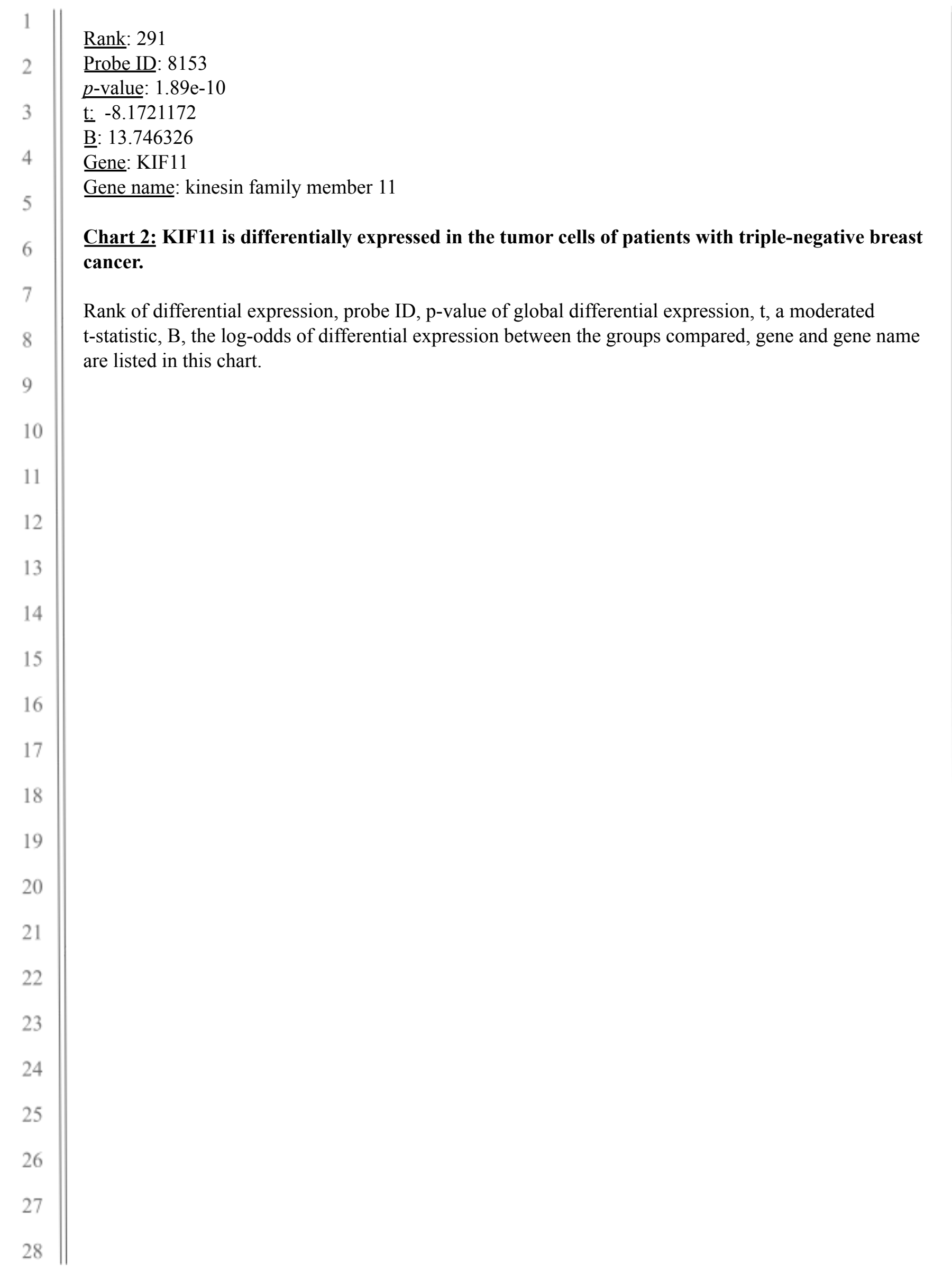




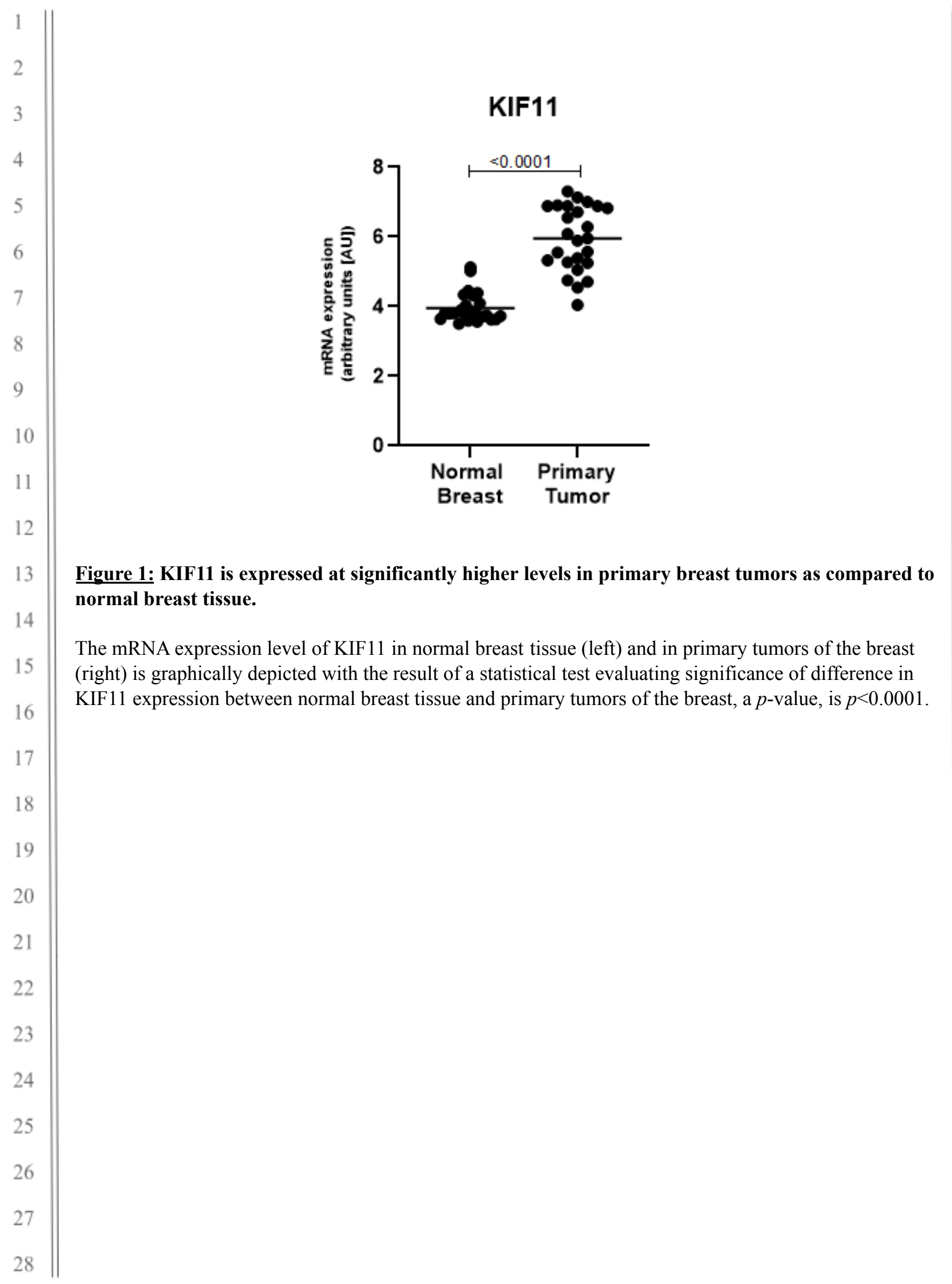




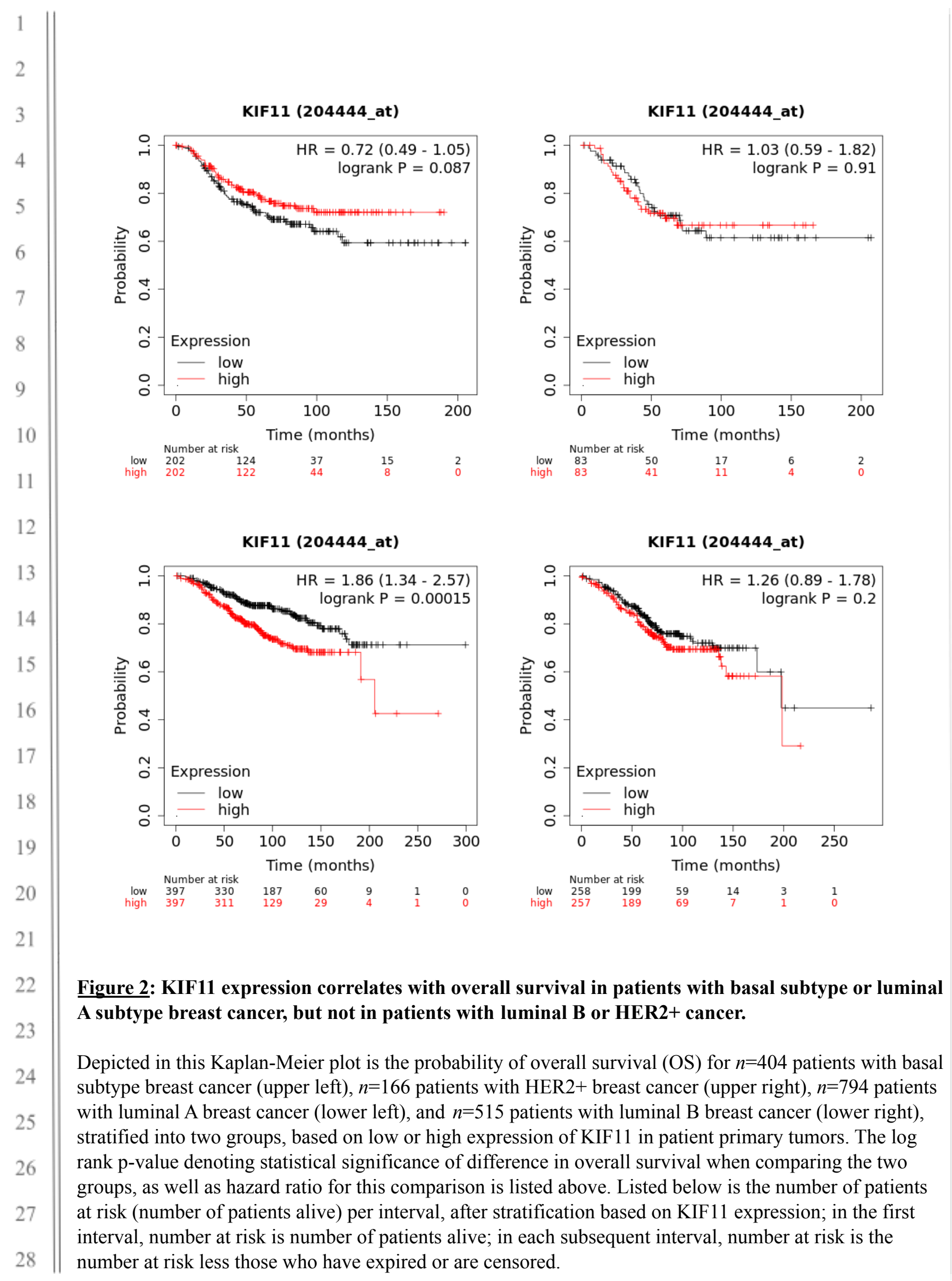




\begin{tabular}{l||l}
1 & Basal \\
2 & Low KIF11 expression: 52.92 months \\
3 & High KIF11 expression: 75.37 months \\
4 & Luminal A \\
5 & Low KIF11 expression: 175.46 months \\
6 & High KIF11 expression: 95.04 months
\end{tabular}

Chart 3: In basal subtype breast cancer, median overall survival is superior in patients with high primary tumor expression of KIF11, while in luminal A subtype breast cancer, median overall survival is superior in patients with low primary tumor expression of KIF11.

The overall survival of $n=404$ basal subtype breast cancer patients and $n=794$ luminal A subtype breast cancer patients based on stratification into low or high tumor expression of KIF11 is listed in this chart. 